\title{
Plasma-assisted oxide removal from ruthenium-coated EUV optics
}

A. Dolgov, C. J. Lee, F. Bijkerk, A. Abrikosov, V. M. Krivtsun, D. Lopaev, O. Yakushev, and M. van Kampen

Citation: Journal of Applied Physics 123, 153301 (2018); doi: 10.1063/1.5006771

View online: https://doi.org/10.1063/1.5006771

View Table of Contents: http://aip.scitation.org/toc/jap/123/15

Published by the American Institute of Physics

\section{Articles you may be interested in}

Thrust performance, propellant ionization, and thruster erosion of an external discharge plasma thruster Journal of Applied Physics 123, 153302 (2018); 10.1063/1.5023829

Terahertz radiation generation through the nonlinear interaction of Hermite and Laguerre Gaussian laser beams with collisional plasma: Field profile optimization

Journal of Applied Physics 123, 153101 (2018); 10.1063/1.5019430

The electrical resistivity of rough thin films: A model based on electron reflection at discrete step edges Journal of Applied Physics 123, 155107 (2018); 10.1063/1.5020577

Effect of capping layer on spin-orbit torques

Journal of Applied Physics 123, 153901 (2018); 10.1063/1.5023670

Investigating the evolution of local structure around $\mathrm{Er}$ and $\mathrm{Yb}$ in $\mathrm{ZnO}: \mathrm{Er}$ and $\mathrm{ZnO}: \mathrm{Er}, \mathrm{Yb}$ on annealing using $\mathrm{X}$ ray absorption spectroscopy

Journal of Applied Physics 123, 153102 (2018); 10.1063/1.5022638

Atomically thin transition metal layers: Atomic layer stabilization and metal-semiconductor transition Journal of Applied Physics 123, 154301 (2018); 10.1063/1.5024200

\section{PHYSICS TODAY}

WHITEPAPERS
MANAGER'S GUIDE

Accelerate R\&D with

Multiphysics Simulation

\section{READ NOW}

PRESENTED BY

И $\subset$ OMSOL 


\title{
Plasma-assisted oxide removal from ruthenium-coated EUV optics
}

\author{
A. Dolgov, ${ }^{1}$ C. J. Lee, ${ }^{1,2}$ F. Bijkerk, ${ }^{1}$ A. Abrikosov, ${ }^{3}$ V. M. Krivtsun, ${ }^{3}$ D. Lopaev, ${ }^{4}$ \\ O. Yakushev, ${ }^{3}$ and M. van Kampen ${ }^{5}$ \\ ${ }^{1}$ XUV Optics Group, University of Twente, MESA+ Institute for Nanotechnology, Enschede, The Netherlands \\ ${ }^{2}$ Insitute of Engineering, Hogescholen Fontys, Eindhoven, The Netherlands \\ ${ }^{3}$ Institute for Spectroscopy RAS, Moscow, Russia \\ ${ }^{4}$ Skobeltsyn Institute for Nuclear Physics, Moscow State University, Moscow, Russia \\ ${ }^{5}$ ASML, Veldhoven, The Netherlands
}

(Received 27 September 2017; accepted 24 March 2018; published online 18 April 2018)

\begin{abstract}
An experimental study of oxide reduction at the surface of ruthenium layers on top of multilayer mirrors and thin $\mathrm{Ru} / \mathrm{Si}$ films is presented. Oxidation and reduction processes were observed under conditions close to those relevant for extreme ultraviolet lithography. The oxidized ruthenium surface was exposed to a low-temperature hydrogen plasma, similar to the plasma induced by extreme ultraviolet radiation. The experiments show that hydrogen ions are the main reducing agent. Furthermore, the addition of hydrogen radicals increases the reduction rate beyond that expected from simple flux calculations. We show that low-temperature hydrogen plasmas can be effective for reducing oxidized top surfaces. Our proof-of-concept experiments show that an in situ, EUV-generated plasma cleaning technology is feasible. Published by AIP Publishing. https://doi.org/10.1063/1.5006771
\end{abstract}

\section{INTRODUCTION}

Photolithographic technology, employing extreme ultraviolet (13.5 nm wavelength) radiation, has seen significant progress recently. ${ }^{1}$ Multilayer mirror (MLM) optics, comprised of 50-60 bi-layers of Mo:Si, each layer being $6.7 \mathrm{~nm}$ thick, are used in this spectral range. To maximise the lifetime of such optics, oxidation of the top protective coating under the intensive EUV radiation ${ }^{2}$ should be prevented. Oxidation can be controlled by coating the mirrors with few nm of ruthenium.

Water molecules, which are physically adsorbed on the ruthenium surface, absorb EUV photons. This may either partially or fully dissociate the water, leading to the formation of atomic oxygen. Atomic oxygen generation allows oxidation to proceed during EUV radiation. It is noteworthy that electron irradiation at similar energies $(\sim 100 \mathrm{eV})$ does not result in such an intense Ru oxidation. ${ }^{3}$

To control oxidation, a technology that efficiently and precisely cleans MLM surfaces must be available. The contamination and cleaning of EUV optics have been the subject of intense research recently. ${ }^{4-8}$ Atomic hydrogen is currently applied to remove different types of contaminations. ${ }^{5,9,10}$ However, atomic hydrogen is not a very effective agent for ruthenium oxide reduction. ${ }^{10}$ In the case of exposure to highenergy photons, photoionisation prevents the formation of a high-density flux of $\mathrm{H}$ atoms toward the irradiated surface. It imposes considerable limitations on the cleaning speed and selectivity. On the other hand, plasma sources are known to efficiently reduce oxide layers from many surfaces. ${ }^{11-13}$ However, these plasmas are typically high-density, hightemperature plasmas and, as such, are incompatible with EUV mirrors.

An alternative is to use the plasma generated by ionization due to EUV radiation. The photon energy of EUV radiation is $92 \mathrm{eV}$, sufficient to ionize hydrogen. Thus, direct photo-ionization of the background gas, along with secondary ionization due to electrons that are emitted from MLMs, creates a short-lived plasma above the mirror surface, which may clean these surfaces in-line. ${ }^{14,15}$

Earlier works ${ }^{16}$ have proven that carbon can be efficiently etched from multilayer mirrors in a EUV-induced hydrogen plasma. This suggests that in-line cleaning could also work for surface oxides as well.

In the absence of EUV, the rates of Ru oxidation and reduction are governed by two rate equations,

$$
\begin{aligned}
\mathrm{d}\left[\mathrm{N}_{\mathrm{RuOx}}\right] / \mathrm{dt} & =\mathrm{k}_{1}\left[\mathrm{~N}_{\mathrm{H} 2 \mathrm{O}}\right]\left[\mathrm{N}_{\mathrm{Ru}}\right]-\mathrm{k}_{2}\left[\mathrm{~N}_{\mathrm{H} 2}\right]\left[\mathrm{N}_{\mathrm{RuOx}}\right], \\
\mathrm{d}\left[\mathrm{N}_{\mathrm{Ru}}\right] / \mathrm{dt} & =\mathrm{k}_{2}\left[\mathrm{~N}_{\mathrm{H} 2}\right]\left[\mathrm{N}_{\mathrm{RuOx}}\right]-\mathrm{k}_{1}\left[\mathrm{~N}_{\mathrm{H} 2 \mathrm{O}}\right]\left[\mathrm{N}_{\mathrm{Ru}}\right],
\end{aligned}
$$

where $\mathrm{N}_{\mathrm{Ru}}, \mathrm{N}_{\mathrm{RuO}_{\mathrm{x}}}, \mathrm{N}_{\mathrm{H}_{2}}$, and $\mathrm{N}_{\mathrm{H}_{2} \mathrm{O}}$ numbers are surface densities for $\mathrm{Ru}, \mathrm{RuO}_{\mathrm{x}}, \mathrm{H}_{2}$, and $\mathrm{H}_{2} \mathrm{O}$ (the latter two being the main reducing and oxidising agents). $k_{1}$ and $k_{2}$ are effective rate coefficients of $\mathrm{Ru}$ oxidation and reduction from $\mathrm{RuO}_{\mathrm{x}}$ by hydrogen. Furthermore, the ratios of the number densities depend on the partial pressures of molecular hydrogen and water in a gas phase and desorption temperatures of these species. Hydrogen desorption starts from clean ruthenium $\mathrm{Ru}(0001)$ at $400 \mathrm{~K}$ and for oxidized surface $\mathrm{RuO}_{2}(110)$ at $300 \mathrm{~K}$, while water desorption starts from ruthenium $\mathrm{Ru}(0001)$ at $150 \mathrm{~K}$ and for oxidized surface $\mathrm{RuO}_{2}(110)$ at $200 \mathrm{~K} .{ }^{5,17}$ Since the partial pressure of at least one of these chemical agents $\left(\mathrm{H}_{2}, \mathrm{H}_{2} \mathrm{O}\right)$ can be controlled in most vacuum systems, the surface coverage is often, to first order, under experimental control.

However, although the number densities of the background gases are under control, the gases and surface adsorbed species are often partially dissociated or ionized. For instance, on a $\mathrm{Ru}$ surface, a fraction of the adsorbed water partially dissociates. Absorption of EUV leads to the formation of oxygen radicals, vastly increasing the rate of oxide formation. On the other hand, hydrogen also dissociates on $\mathrm{Ru}$, forming more active radical species, while the presence of EUV leads to a large radical and ion flux incident on the surface, changing the 
rate at which reduction occurs. Assuming linearity (e.g., that all rates are independent of each other), these processes can be taken into account in composite rates, $k_{1}$ and $k_{2}$. The rates $k_{1}$ and $k_{2}$ must be known, and the major contributors to the composite rate understood in order to control the balance between oxidation and reduction.

Although oxidation of ruthenium surfaces has been described by a model, studies of reduction and oxidation are limited. ${ }^{18}$ Specifically, the reduction of the surface, and the balance between oxidation and reduction have not been studied in detail. Furthermore, the balance between the oxidation and reduction under EUV radiation, and, consequently, in the presence of ions and radical species has not been investigated.

The aim of this paper is to present an analysis of the main mechanisms for oxide reduction. Thus, the potential for balancing oxidation and reduction through the use of EUVgenerated active species (ions and radicals) can be evaluated. Specifically, the possibility for in-line cleaning, via the lowpressure hydrogen plasma that is induced by EUV radiation, is considered.

We present new data on the oxidation and reduction of the top ruthenium surface of MLMs in the presence of EUV radiation. A low-density, inductively coupled, plasma and a surface wave discharge (SWD) plasma were used to determine the absolute etch rates due to hydrogen ions, radicals, and the combination of the two. We conclude that, under EUV lithography relevant conditions, efficient oxide removal is possible using in-line plasma cleaning.

\section{OXIDIZED RUTHENIUM SAMPLES}

In order to relate observations of oxidation and reduction on Ru-terminated multilayer mirrors to more fundamental processes, we also performed experiments on model systems.

Ruthenium-terminated multilayers were studied in EUV exposures, where the response of the mirror plays an important role in the formation of the plasma near the mirror surface. The MLM samples consisted of Mo/Si multilayers $(25 \times 25 \mathrm{~mm})$, terminated with $<3 \mathrm{~nm}$ of ruthenium. EUV-induced ruthenium oxidation/reduction processes were studied under a variety of exposure conditions and partial pressures of hydrogen and water. We chose experimental conditions that are as close as possible to those relevant to EUV lithography (EUVL).

For model-system studies, the samples consisted of plasma-oxidized ruthenium films, which are structurally and chemically similar to ruthenium oxide films that grow under EUV illumination. ${ }^{5}$ The deposited Ru layer was $20 \mathrm{~nm}$ thick, deposited on the oxide-terminated surface of a Si wafer. The $\mathrm{Ru}$ layer was subsequently oxidized by exposure to an oxygen plasma (see below for details on the oxide layer thickness). The reduction of the oxide layer was performed in a plasma reactor that produces ion energies and fluxes similar to those produced by EUV-induced plasmas. This model system is more straightforward to analyze than a regular MLM. Furthermore, by using a relatively thick Ru layer, the samples could also be used as electrodes to measure the ion flux.

For MLM studies, the thickness of the oxide layer was measured in-situ, using ellipsometry. To ensure the accuracy of the optical model, thickness measurements derived from Energy Dispersive Spectroscopy (EDS) and X-ray fluorescence analysis (XRF) were compared with ellipsometry estimates. Figures 1(a) and 1(b) illustrate the principle for an $\mathrm{Ru} / \mathrm{Si}$ sample. Figure 1(b) shows a typical calibration curve for ellipsometry and XRF. The symbols show the intensity of the EDS and the model-predicted ruthenium oxide thickness, based on measured ellipsometry data. The model uses a modified set of optical constants obtained from Palik. ${ }^{19}$ The oxide layers for the samples (Fig. 1) exposed to oxygen plasma were found to have thicknesses of $0.26 \mathrm{~nm}, 0.48 \mathrm{~nm}$, and $0.66 \mathrm{~nm}$. These thicknesses correspond to less than 1 monolayer $(\mathrm{ml})$ of oxide, more than $1 \mathrm{ml}$ and less than $2 \mathrm{ml}$ of oxide, and more than $2 \mathrm{ml}$ of oxide, respectively, assuming $\mathrm{RuO}_{2}(110)$ oxide structure. Three thicknesses of oxide film were chosen so that the influence of oxide depth on oxide reduction could be studied.

A linear regression of the data results in a measurement accuracy of $\pm 0.05 \mathrm{~nm}$ for ellipsometry. For the plasmaassisted reduction experiments, only ex-situ EDS measurements were performed.

\section{EVOLUTION OF OXIDATION AND REDUCTION OF RUTHENIUM IN WATER/HYDROGEN MEDIUM}

EUV-induced oxidation and reduction was -studied with a EUV exposure tool using Ru-terminated MLM samples. A schematic representation of the first used EUV set-up is given in Fig. 2. The tool consists of two main parts: the EUV source and the exposure chamber. The exposure chamber is separated from the radiation source by a spectral-purity filter (SPF) which ensures that the sample is only exposed to EUV light. The SPF is a free-standing SiZr filter with $50 \mathrm{~nm}$ thickness and

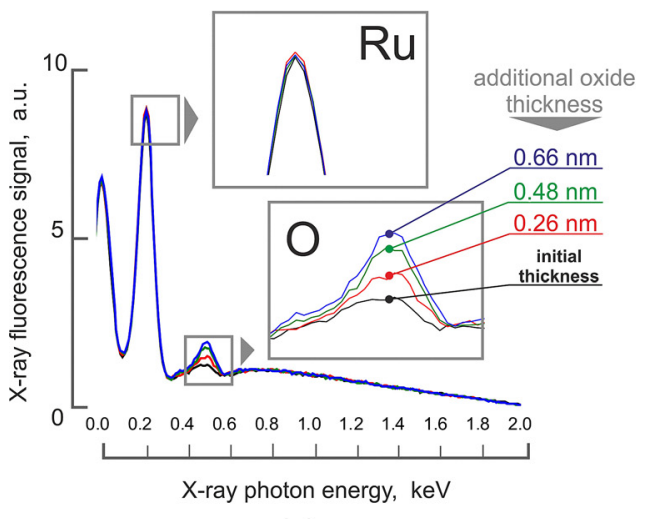

(a)

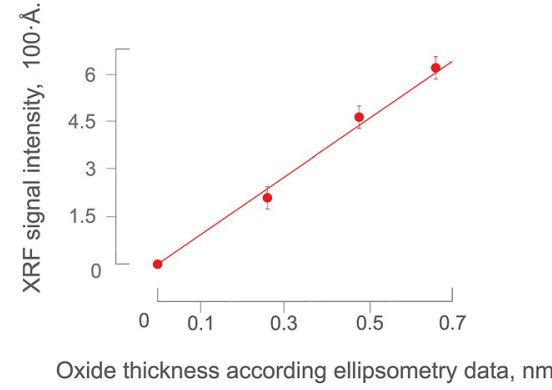

(b)
FIG. 1. X-ray fluorescence analysis of the oxidized $20 \mathrm{~nm}$ thick Ru film on $\mathrm{Si}$. The presence of an oxygen peak $(525 \mathrm{eV})$ shows that the ruthenium surface is always oxidized in the case of ex-situ measurements. For our experiments, the initial oxide thickness is less than one monolayer (a) and calibration curve for ellipsometry and XRF is linear (b). 


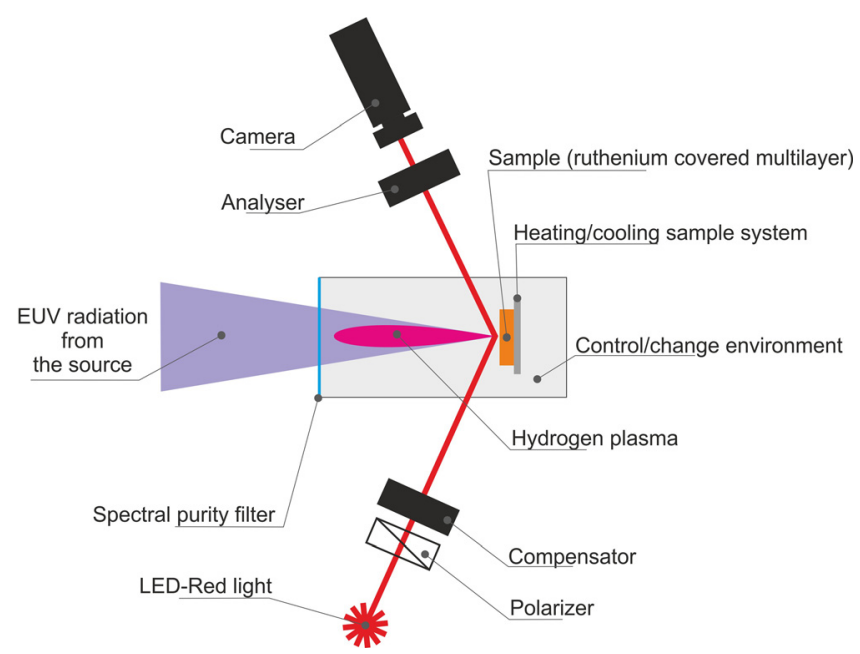

FIG. 2. EUV exposure tool.

$78 \%$ in-band transmission. The EUV beam (FWHM $\sim 4 \mathrm{~mm}$ ) was focused to a $2 \mathrm{~mm}$ diameter spot with an average power density of $5.7 \mathrm{~mW} / \mathrm{mm}^{2}$ at $600 \mathrm{~Hz}$ during 35 million pulses.

Within the exposure chamber, samples are irradiated at the focus of the EUV beam under varying background gas pressures and sample temperatures. The base pressure of the system after bake-out is $4 \times 10^{-8} \mathrm{mBar}$. Water and hydrogen are introduced to the exposure chamber via two separate valves. For in-situ oxidation/reduction studies, the natural oxide layer due to exposure to atmosphere was first removed by hydrogen radical etching within the load lock chamber.

While the sample is being irradiated, an imaging ellipsometer allows for in-situ monitoring of changes to the sample surface (e.g., growth or reduction of surface oxide) with sub$\mathrm{nm}$ sensitivity; with this technique, the oxidation and reduction of the surface can be spatially and temporally resolved.

The imaging ellipsometer was developed in-house. It is a rotating compensator ellipsometer in a polarizer-compensatorsample-analyzer configuration. It uses a $\sim 630 \mathrm{~nm}$ LED light source that is collimated to a $\sim 2.5 \mathrm{~cm}$ diameter beam that is incident at a 70 degree angle on the sample. The reflected light is detected by a $2 / 3^{\prime \prime} \mathrm{CCD}$ camera with a $75 \mathrm{~mm}$ focal length lens. The timing of the camera trigger is synchronized to the rotation of the compensator, allowing one to determine $\Psi$ (the amplitude ratio upon reflection) and $\Delta$ (phase shift) for each pixel.

Samples were held at a fixed temperature using a closed cycle system (Phoenix II, Thermoscientific). The minimum sample temperature is limited by the thermal contact between the cooling circuit and the MLM. The actual temperature is measured by a small $(2 \times 2 \mathrm{~mm})$ PT1000 resistive temperature sensor $\left(3.8 \Omega /{ }^{\circ} \mathrm{C}\right)$ that is clamped on the metallic frame that holds the mirror.

An example of the oxide thickness determination, as extracted from the optical constants, is presented in Fig. 3.

In order to illustrate control for oxidation and reduction of the MLM protective layer, we placed the samples under the EUV radiation and monitored the oxide thickness under different $\mathrm{H}_{2} \mathrm{O}: \mathrm{H}_{2}$ ratios using ellipsometry. Figure 4 shows the evolution of $\mathrm{Ru}$ oxidation and reduction as the partial

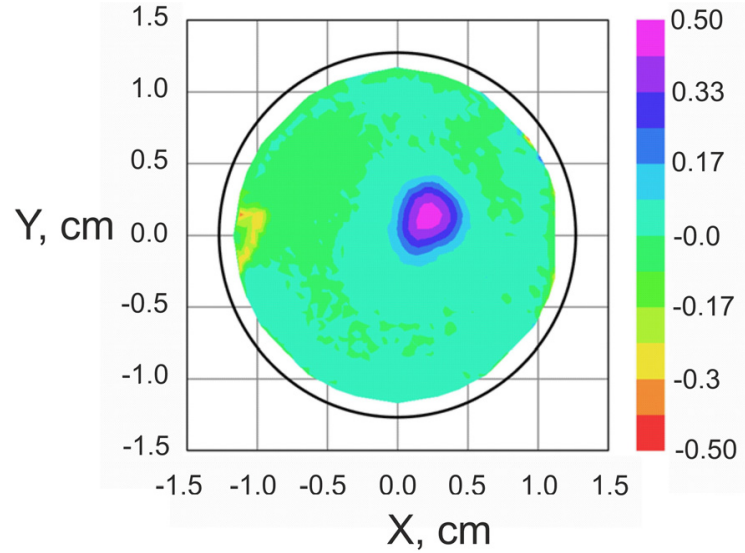

$\mathrm{nm}$

FIG. 3. Ruthenium oxide thickness map. The sample was oxidized at $2 \times 10^{-5} \mathrm{mBar}$ of partial water pressure and $4 \times 10^{-8} \mathrm{mBar}$ of partial hydrogen pressure. Surface temperature was $8^{\circ} \mathrm{C}$. The EUV power density was $5.7 \mathrm{~mW} / \mathrm{mm}^{2}$ at $600 \mathrm{~Hz}$, and the total number of EUV pulses was $35 \times 10^{6}$.

pressures of water and hydrogen are varied. The $\mathrm{RuO}_{\mathrm{x}}$ thickness and phase shift $(\Delta$-signal) are plotted as a function of exposure time. The experiment began with a sample that was oxidized to a depth of $0.12 \mathrm{~nm}$. First, the ability to obtain a balance between oxidation and reduction is demonstrated by exposing the sample to a mixture of water and hydrogen (partial pressures of $\mathrm{P}_{\mathrm{H}_{2} \mathrm{O}}=2 \times 10^{-5} \mathrm{mBar}$ and $\mathrm{P}_{\mathrm{H}_{2}}=5 \times 10^{-2}$ $\mathrm{mBar}$ at a temperature of $4{ }^{\circ} \mathrm{C}$ ). Here, to within our measurement accuracy, an equilibrium is observed: oxidation is balanced by oxide reduction.

Once the partial pressure of water is reduced to background levels ( $\sim 10^{-8} \mathrm{mBar}$ ) (30 min-64 min, Fig. 4), reduction dominates. In this case, $\Delta$ increases, indicating the reduction of the oxide thickness from 0.12 to $0.08 \mathrm{~nm}$. We also demonstrate that this can be reversed $(64 \mathrm{~min}-140 \mathrm{~min}$, Fig. 4) by reducing the hydrogen partial pressure to background levels and returning the water partial pressure to $\mathrm{P}_{\mathrm{H}_{2} \mathrm{O}}=2 \times 10^{-5} \mathrm{mBar}$. Under these conditions, as expected, $\Delta$ reduces, indicating oxidation of the $\mathrm{Ru}$.

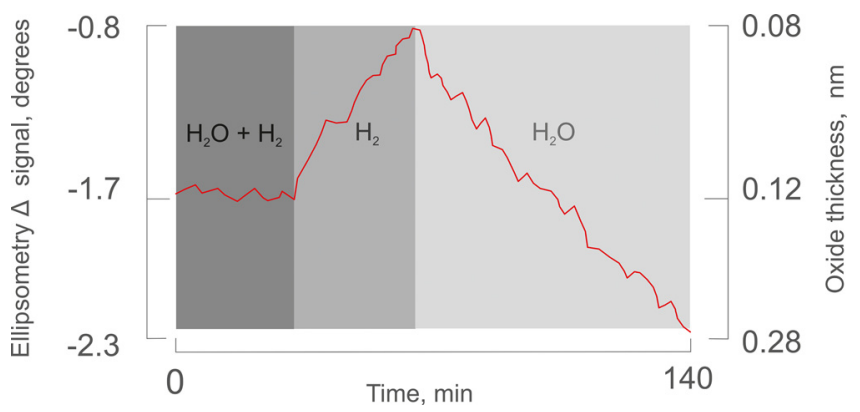

FIG. 4. In-situ oxide thickness as a function of time when varying the partial pressures of water and hydrogen. The change in ellipsometry phase shift signal $(\Delta)$, which is approximately linear with oxide thickness in this range, is used as an approximation for oxide thickness. The sample, oxidized to a thickness of $0.12 \mathrm{~nm}$, is first exposed to a balanced water $\left(\mathrm{P}_{\mathrm{H}_{2} \mathrm{O}}=2 \times 10^{-5}\right.$ $\mathrm{mBar})$ and hydrogen $\left(\mathrm{P}_{\mathrm{H}_{2}}=5 \times 10^{-2} \mathrm{mBar}\right)$ environment. Then, a hydrogendominant environment $\left(\mathrm{P}_{\mathrm{H}_{2}}=5 \times 10^{-2} \mathrm{mBar}, \mathrm{P}_{\mathrm{H}_{2} \mathrm{O}}<2 \times 10^{-8} \mathrm{mBar}\right)$ and finally to a water-dominant environment $\left(\mathrm{P}_{\mathrm{H}_{2} \mathrm{O}}=2 \times 10^{-5} \mathrm{mBar}, \mathrm{P}_{\mathrm{H}_{2}}<5\right.$ $\left.\times 10^{-8} \mathrm{mBar}\right)$. For all measurements, the sample temperature was maintained at $4{ }^{\circ} \mathrm{C}$. 
The details of the mechanisms underlying the balance between oxidation and reduction require a separate study and is not the subject of the current paper. Here, we focus on an empirical model that describes the balance.

The rate of oxide reduction and growth, obtained for $\mathrm{H}_{2}$ and $\mathrm{H}_{2} \mathrm{O}$, is very close to the absolute value, suggesting a first-order approximation

$$
\mathrm{dz} / \mathrm{dt}=\alpha_{\mathrm{r}}\left(\mathrm{P}_{\mathrm{H}_{2} \mathrm{O}}, \mathrm{P}_{\mathrm{H}_{2}}\right)+\alpha_{\mathrm{ox}}\left(\mathrm{P}_{\mathrm{H}_{2} \mathrm{O}}, \mathrm{P}_{\mathrm{H}_{2}}\right),
$$

where $z$ is the oxide thickness and $\alpha$ is the oxide/reduction rate (slope of the line at Fig. 4). In case of reduction, slope $\boldsymbol{\alpha}_{\mathbf{r}}$, is considered to be positive $(0.21 \pm 0.1 \mathrm{~nm} / \mathrm{h}$ from Fig. 4). Oxidation rate $\alpha_{\mathbf{o x}}$ is negative ( $-0.17 \pm 0.1 \mathrm{~nm} / \mathrm{h}$ from Fig. 4).

The validity of Eq. (1) was checked directly by comparing measured to predicted oxide thickness growth rates at varying partial pressures of hydrogen and water. Figure 5 shows the measured oxidation/reduction rates for water/hydrogen presence only and experimental data for the case of presence of both water and hydrogen. The total $\mathrm{d} z / \mathrm{d} t$ rate from (1) for the $2 \times 10^{-5} \mathrm{mBar}_{2} \mathrm{O}$ environment pressure at different partial pressure of hydrogen was calculated using data for pure hydrogen $\left(\alpha_{\mathrm{r}}\right)$ and pure water $\left(\alpha_{\mathrm{ox}}\right)$ environments. The experimental value for the total rate is in good agreement with that calculated from the first-order approximation.

It can also be seen (see Fig. 4) that with increasing hydrogen pressure, oxygen removal is sufficiently fast to avoid Ru oxidation.

In summary, we observed that, under EUV radiation, the oxidation and reduction rates are only dependent on their respective feed-gases rates. It is worth noting that the experiments were carried out at relatively low temperatures $\left(4^{\circ} \mathrm{C}\right)$ in order to enhance the oxidation process. At a higher temperature, oxidation at $\mathrm{P}_{\mathrm{H}_{2} \mathrm{O}}=2 \times 10^{-5} \mathrm{mBar}$ is not observed. The physical mechanism of this process should be determined; however, we assume that the main factors could be surface activation of ruthenium or carbon growth. With increasing

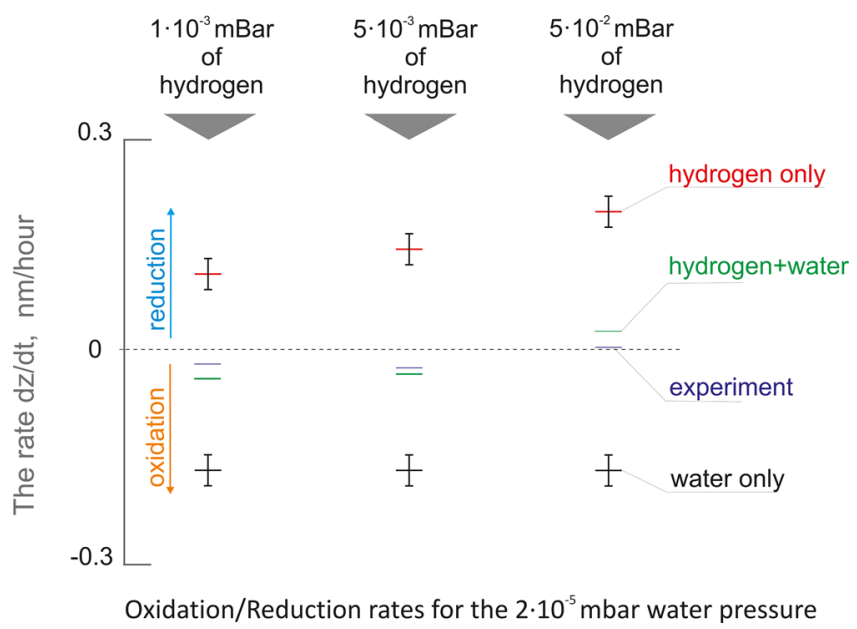

FIG. 5. Oxidation rate, $\alpha_{\mathrm{ox}}$ (black), for constant $\mathrm{H}_{2} \mathrm{O}$ pressure $\left(2 \times 10^{-5}\right.$ mbar) only and reduction rate, $\alpha_{\mathrm{r}}$ (red), for different partial pressures of $\mathrm{H}_{2}$ only (shown at the top of the figure). Observed total dz/dt rate marked with blue color (experiment) and, predicted total dz/dt rate (just sum of $\mathrm{H}_{2}$ and $\mathrm{H}_{2} \mathrm{O}$ rates) marked with green for different partial pressures of hydrogen and a fixed partial pressure of water. hydrogen partial pressure, hydrogen ions will be formed into the exposure chamber. The high energy EUV-photons ionize background gaseous hydrogen as well as the hydrogen molecules adsorbed on the surface. Secondary electrons significantly influence this process. One can assume that the lowpressure EUV-induced hydrogen plasma that formed above the sample is primarily responsible for ruthenium reduction.

\section{PLASMA-ASSISTED RUTHENIUM REDUCTION}

To study the plasma-assisted Ru oxide reduction mechanisms, we performed experiments on a model system: $20 \mathrm{~nm}$ $\mathrm{Ru}$, deposited on a Si wafer (all experiments of this Section) in a hydrogen discharge plasma with conditions that closely reproduce those of a EUV-induced plasma.

Samples with varying thicknesses of ruthenium oxide were placed in a surface wave discharge plasma reactor, shown schematically in Fig. 6. The samples were placed along a long quartz tube with an inner diameter of $55 \mathrm{~mm}$, where a low-pressure low-density $\mathrm{H}_{2}$ plasma is generated. ${ }^{14-16}$ The plasma is generated in pure hydrogen, at a pressure of 100 mTorr $(13.3 \mathrm{~Pa})$, using an electrode-less surface wave discharge (SWD) with a power of $\sim 10-12 \mathrm{~W}$, and oscillating at a frequency of $40 \mathrm{MHz}$. Due to a surface electromagnetic wave, a quasi-neutral plasma column is produced in the tube beyond the SWD antenna. The electron temperature is nearly constant along the plasma column, while the plasma density gradually drops off as the distance from the antenna increases. ${ }^{16}$ These characteristics mean that the plasma density and the corresponding ion flux vary by up to two orders of magnitude, depending on the sample position, while the ion energy spectrum remains the same for all samples. The ion flux incident on the sample surfaces was, for each sample position, derived from the analysis of IV curves for flat Langmuir probes. ${ }^{16}$ To vary the energy of the incident ion flux, the sample holders were negatively biased. Owing to the fast ion conversion reaction: $\mathrm{H}_{2}^{+}+\mathrm{H}_{2} \rightarrow \mathrm{H}_{3}^{+}+\mathrm{H}\left(k=1.5 \times 10^{-9} \mathrm{~cm}^{3} / \mathrm{s}\right)$, the dominate ion is $\mathrm{H}_{3}{ }^{+}$. $14-16,20,21$

One major difference between the generated plasma described above and the EUV-induced plasma is that the latter consists of a mixture of hydrogen radicals and ions, while the

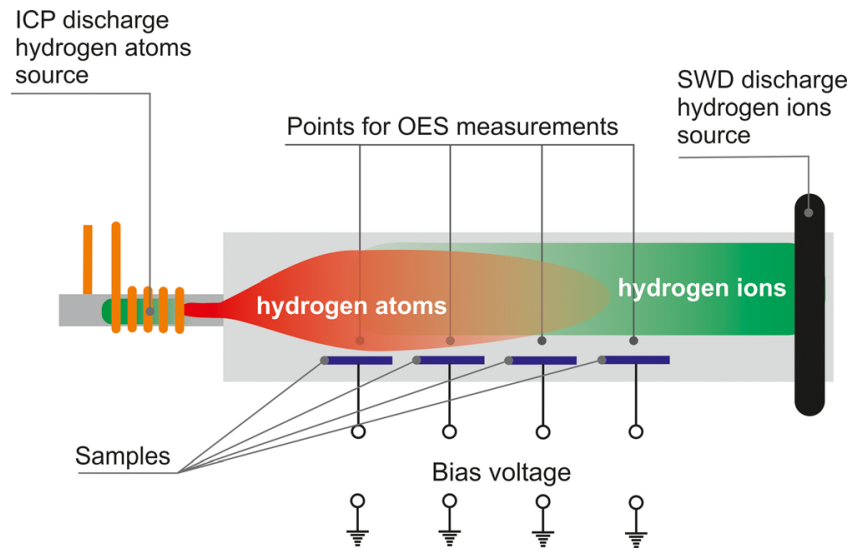

FIG. 6. The SWD plasma source (right hand side) generates ions with a constant energy spectrum but decreasing density. The ICP plasma reactor (lefthand side) was used to generate $\mathrm{H}$ atoms. As a result, the different sample positions are subjected to different ratios of ions to radicals. 
radical component of the former is negligible. To generate radicals, a flow of molecular hydrogen passes through the smaller diameter quartz tube, where it is dissociated by an inductively coupled plasma (ICP) discharge (13.56 MHz, $200 \mathrm{~W})$. Under the given discharge conditions, up to $\sim 40 \%$ of molecular hydrogen is dissociated. However, $\mathrm{H}$ atom recombination, mainly at the surfaces of the metallic flanges that couple the quartz tubes ${ }^{22}$ is rapid, so only about $1 \%-2 \%$ of atomic hydrogen reaches the sample holders. Despite rapid recombination, the atomic hydrogen contribution from the $13.56 \mathrm{MHz}$ discharge is an order of magnitude more than that from the SWD $40 \mathrm{MHz}$ discharge. The density of hydrogen radicals over the sample positions was measured using actinometry. Details of the measurements are presented in the Appendix. To calibrate the actinometry signal, $10 \%$ of argon was added to the hydrogen flow and the difference in the ratio of the emission spectrum lines was used to estimate the density of dissociated hydrogen over the samples. ${ }^{21,22}$ The ion and atomic hydrogen fluxes, incident on the sample surfaces, were calculated from the measured ion and atom densities by using "flat-probe" approach and ideal gas relations, respectively.

Thus, the combination of a low-power SWD plasma with an ICP source for $\mathrm{H}$ atoms production allows for the effects of low-density hydrogen plasma, atomic hydrogen, and the combination of these two, to be studied in a controlled manner.

The rate of $\mathrm{O}$ removal from the oxidized Ru surface was obtained for three cases: samples were treated by $\mathrm{H}$ atoms only; $\mathrm{H}_{3}+$ ions only; and $\mathrm{H}_{3}+$ ions and $\mathrm{H}$ atoms at the same time. To control the energy of the incident ions, the samples were biased with respect to the plasma potential. The oxygen atom removal per incident ion was estimated for different sample bias voltages and ion fluxes. The parameters of the plasma above the sample surface were determined, based on measured sample voltage-current characteristics. ${ }^{16}$

The best reduction effect was observed for the case of joint exposure to hydrogen atoms and ions. Ions are more effective than atoms, and even a low ion flux was observed to increase the removal rate noticeably. The oxygen removal rate by $\mathrm{H}$ atoms only is relatively low (see Figs. 7 and 8). In order to describe the ruthenium reduction phenomenologically, a linear approximation has been used, as it was for C-cleaning in Ref. 16.

We present the following equation for ruthenium reduction rate $R$ :

$$
R=\beta\left(E_{a c t}, T_{S} . .\right) \cdot F_{H}+\xi\left(E_{i}, T_{S}, . .\right) \cdot F_{i},
$$

where $F_{i}$ and $F_{H}$ are the hydrogen ion and atom fluxes, respectively; $\beta\left(E_{a c t}, T_{s}\right)$ is a coefficient that can be defined as the efficiency with which $\mathrm{H}$ atoms remove $\mathrm{O}$ atoms from the surface. $E_{\text {act }}$ is the activation energy of the surface reaction (typically $\sim 0.5 \mathrm{eV}$ ) and $T_{s}$ is the surface temperature. The parameter $\xi\left(E_{i}, T_{s}\right)$ is a dimensionless coefficient for ions, defined as the efficiency with which $\mathrm{H}_{3}{ }^{+}$ions removes $\mathrm{O}$ atoms from the surface. $\xi\left(E_{i}, T_{s}\right)$ and $\beta\left(E_{a c t}, T_{s}\right)$ depend on many parameters, including surface temperature and, in the case of $\xi$, the ion energy, $E_{i}$.

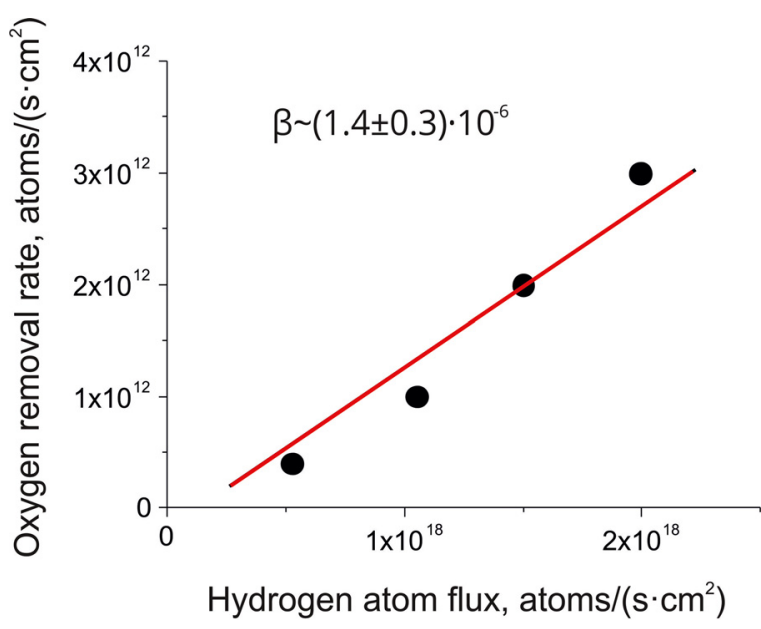

FIG. 7. The dependence of ruthenium reduction rates on atomic hydrogen flux. The per-H-atom efficiency of removing an oxygen atom is estimated from a linear fit to the data.

Of course, such a description does not directly take into account the transport of oxygen from below the surface, the mechanism by which surface vacancies are generated, etc. However, this model is sufficient to predict in-situ reduction of $\mathrm{Ru}$ under conditions that are close to those applied in EUVL. In EUVL, the goal is to keep the oxide layer as thin as possible, so reduction reactions of ultrathin layers are dominated by surface reactions, rather than diffusion to the surface. Furthermore, all the governing physics and chemistrysurface vacancies, for example-are absorbed into the empirically determined rates.

Figure 7 shows the dependence between the oxygen removal rate and the flux of hydrogen atoms. As expected, the oxygen removal rate increases with increasing hydrogen atom flux. Furthermore, in the range of fluxes investigated here, the oxygen removal rate is approximately linear. The efficiency of a single hydrogen atom removing an oxygen atom is estimated to be $\beta \approx(1.4 \pm 0.3) \times 10^{-6}$ from the slope of a linear fit to the experimental data from Fig. 7.

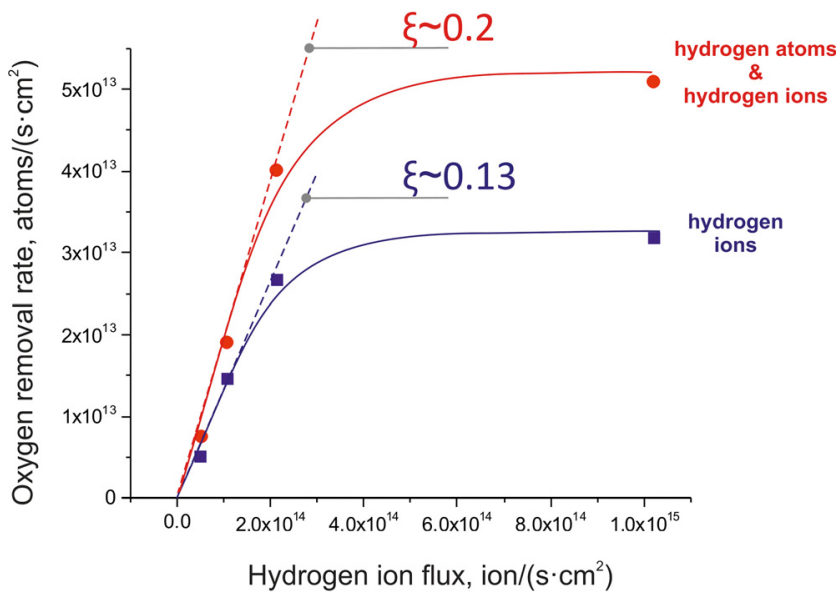

FIG. 8. Effectiveness of oxygen removal from grounded samples. The ruthenium reduction rate was measured for the following cases: surface wave discharge is ON for ion exposure (ions only); and surface wave and ICP discharges are switched onto obtain a mixture of atoms and ions. The initial slopes (slopes of dashed lines) were used to define the per-ion O removal rates. 
Figure 8 shows the oxygen removal rate for the case of ions, and for the case of a mixture of radicals and ions. The slope of the non-saturated part of the curve is estimated from a linear fit to the data. For the grounded samples, the efficiency, $\xi$, was found to be $\sim 0.13$, and somewhat larger $(\sim 0.2)$ for a mixture of radicals and ions. Clearly, ions are more effective than hydrogen radicals alone. This can also be seen by comparing the absolute fluxes of ions and radicals. The ion flux is four orders of magnitude less than for radicals (see Fig. 7), while the reduction rate for ions is still an order of magnitude more than for radicals alone.

To compare the effectiveness of reduction under various conditions, we estimate the average number of oxygen atoms removed by a single hydrogen ion, which we refer to as reduction efficiency, $\xi$. The dependence of $\xi$ on ion energy, shown in Fig. 9, was estimated by measuring reduction rates on biased samples. It can be seen that $\xi$ rises slowly with increasing ion energy at energies below $\sim 40 \mathrm{eV}$. But for energies greater than $40 \mathrm{eV}, \xi$ begins to increase sharply and reaches a value of more than 2 for ion energies $>100 \mathrm{eV}$.

The sharp increase in efficiency may be due to two different mechanisms to remove oxygen from $\mathrm{RuO}_{\mathrm{x}}$. The first, which we refer to as process-1, is dominant at energies $<40 \mathrm{eV}$, while the second, called process-2, requires ion energies $>40 \mathrm{eV}$. Evidence for the existence of process- 1 and process-2 is presented in Fig. 10.

We propose the following mechanism for the observed energy-dependence for ion-assisted removal of oxygen. For low-energy ions $\left(\mathrm{E}_{\mathrm{i}} \lesssim 40 \mathrm{eV}\right)$ where $\xi<0.5$, ion-surface neutralization, which produces hot $\mathrm{H}$ atoms on the surface, is responsible for $\mathrm{O}$ removal, while for higher energies $\left(E_{i}\right.$ $>50 \mathrm{eV}$ ), chemical sputtering starts to contribute, and eventually dominates. Arrhenius plots for these two mechanisms are shown in Fig. 10. The range of energies in our experiments is too low for classical two-body collision model to be valid. However, during plasma treatment, the surface species absorb certain part of ions energy. Or $k T_{s} \approx \gamma E_{i}$ where $\gamma$ is a coefficient that takes into account energy loss due to collisions, stopping

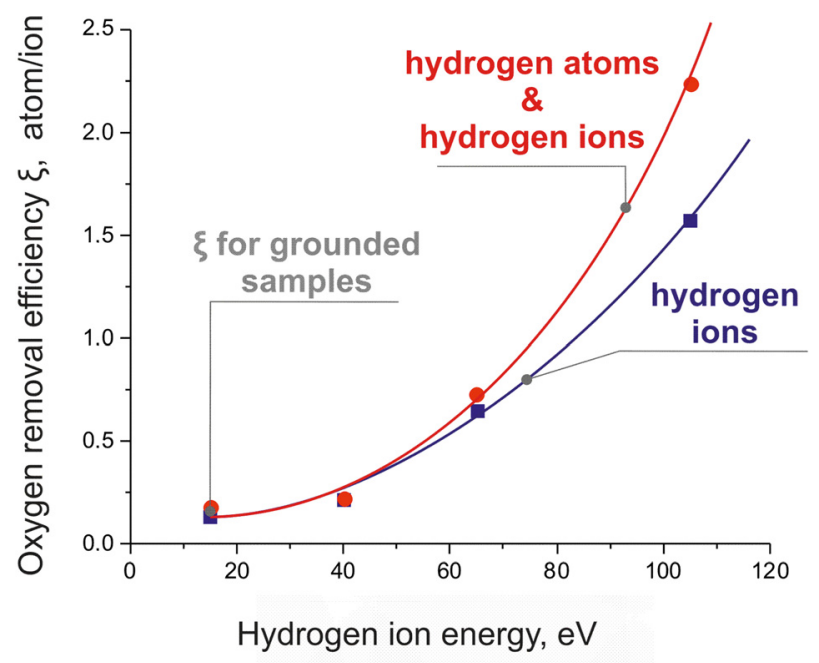

FIG. 9. O atom removal efficiency $\xi$ per ion, as a function of the average ion energy with (red curve) and without (blue curve) additional hydrogen radical flux. processes, etc. Taking this assumption, we fit the dependence between oxygen removal efficiency and $l / E_{i}$ in order to find the activation energies for different ruthenium reduction processes.

As can be seen in Fig. 10, two processes play a role in ruthenium reduction under plasma treatment.

Process- 1 is described by the equation

$$
\ln \xi=\ln A_{1}-1.5 \cdot\left(1 / E_{i}\right),
$$

and dominates at low ion energies, while process-2, which occurs at high ion energies, is given by

$$
\ln \xi=\ln A_{2}-137 \cdot\left(1 / E_{i}\right) .
$$

Given that $k T_{s} \approx \gamma E_{i}$, an estimate for $\gamma$ can be found. The Arrhenius equation becomes

$$
\ln \xi=\ln A_{1}-\left(E_{a} / k T_{S}\right)=\ln A_{1}-\left(E_{a} / \gamma E_{i}\right) .
$$

We propose that process- 1 is the removal of oxygen atoms by hydrogen atoms. The excess energy from the ion dissociates the $\mathrm{RuO}$ bond, making $\mathrm{OH}$ formation highly probable, a process well known from Ref. 10. The activation energy for this process is $0.48 \mathrm{eV},{ }^{10}$ which means that $\gamma=0.32$.

Substituting $\gamma$ into Eq. (4), one finds an activation energy of about $44 \mathrm{eV}$. The energy is higher than the binding energy of surface atoms in oxide structure. This implies that the process responsible for ruthenium reduction is not a direct process. Ions with an energy of $44 \mathrm{eV}$ will, as they stop, drive many energetic processes, such as secondary electron emission, which can dissociate more than a single ruthenium oxide bond. At the same time, these processes result in the formation of atomic hydrogen atoms at or just below the surface of the ruthenium oxide. Moreover, volume vacancies could be generated to increase oxygen diffusion to the upper surface layers. Thus, there are many possible indirect processes that may contribute to process-2. Interestingly, the linearity of process- 2 indicates that only a single pathway is dominant.

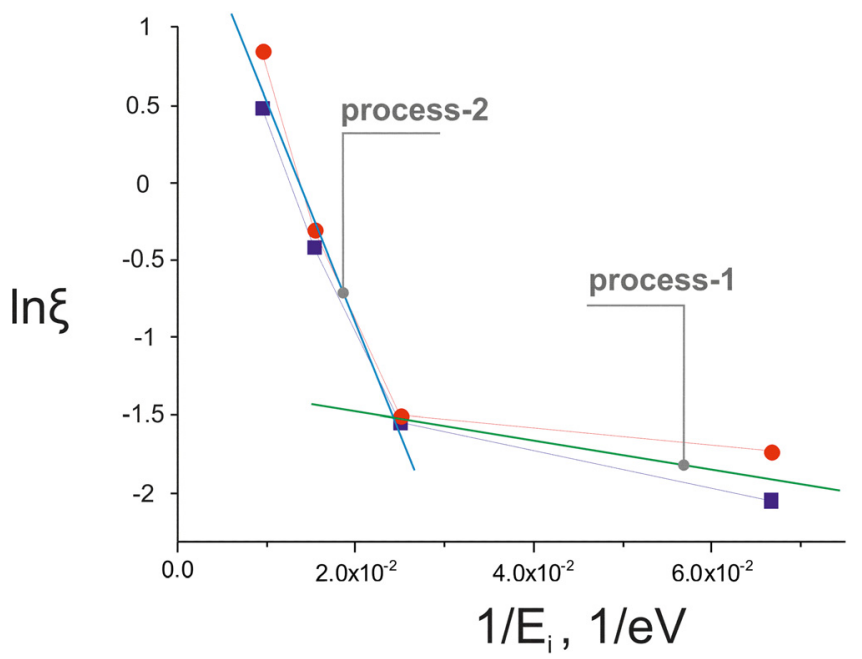

FIG. 10. $\ln \xi$ as a function of $1 / E_{i} \sim 1 / T_{s}$ for ions (blue), ions and radicals (red). As one can see there are two different mechanisms for oxygen removal from $\mathrm{RuO}_{\mathrm{x}}$, which we label process-1 and process-2. Process- 1 is dominant for energies below $40 \mathrm{eV}$, while for energies above $40 \mathrm{eV}$, process-2 becomes increasingly dominant. 
Thus, $\mathrm{H}_{3}{ }^{+}$ions are very effective for removing oxygen from the surface and recovery of $\mathrm{Ru}$ in comparison with $\mathrm{H}$ atoms. However, to evaluate the applicability of in-line plasma etching to EUV lithography, experiments under EUV lithography relevant conditions were performed.

\section{EUV-INDUCED PLASMA EXPERIMENTS}

A second series of EUV exposure experiments were performed in a UHV chamber, attached to a tin discharge produced plasma EUV source, which is described in detail in Ref. 23. Briefly, EUV radiation from a tin plasma is refocused by collector optics at the sample position, located in an UHV chamber. The UHV chamber is separated from the main volume by a spectral purity filter (SPF), which is opaque from the deep UV to the visible range and transmits primarily the source's EUV radiation at a wavelength of $13.5 \mathrm{~nm}$. By using differential pumping and the SPF, the vacuum in the UHV chamber is as low as $10^{-6} \mathrm{~Pa}$. The UHV chamber can be filled with different gases to a pressure up to $100 \mathrm{~Pa}$, and the samples can be biased to control the ion energy of the flux incident on the sample surface.

The EUV beam is focused to a diameter of $12 \mathrm{~mm}$ (FWHM $6.3 \mathrm{~mm}$ ) and has a spatially and temporally averaged intensity of $0.13 \mathrm{~W} / \mathrm{cm}^{2}$ in the focus spot. To eliminate most of the scattered EUV radiation, a diaphragm of synthetic mica $(\emptyset=8 \mathrm{~mm})$ was placed on top of the samples. The EUV source operates at $1.5 \mathrm{kHz}$, and the energy flux per pulse is $0.085 \mathrm{~mJ} / \mathrm{cm}^{2}$ in a $100 \mathrm{~ns}$ (FWHM) pulse.

Ruthenium films of $25 \mathrm{~nm}$ on a silicon substrate $1 \times 1 \mathrm{~cm}^{2}$ size were oxidized in a plasma reactor to a depth of up to $0.69 \mathrm{~nm}$, according to calibrated XRF measurements. Afterwards, they were exposed to EUV in the presence of hydrogen at a pressure of $3 \mathrm{~Pa}$. All exposures were carried out at room temperature with a sample bias voltage of $-100 \mathrm{~V}$.

Three sets of experiments were carried out for 2, 5, and $10 \times 10^{6}$ EUV pulses with MLM samples. The total ion charge, measured by the sample current, was used to estimate both the ion-flux incident on the sample surface, and the number of ions formed in the EUV-induced plasma above the sample. Thereby, the applied radiation doses of 2-, 5-, and $10 \times 10^{6}$ pulses corresponded to an integrated ion dose of 6-, $15-$, and $30 \times 10^{15}$ ions at the surface.

Ruthenium reduction half-profiles for different ion doses (exposure times) are shown in Fig. 11. The hydrogen ion flux profile depends on the EUV intensity profile, which is shown by the line in Fig. 11. It is clearly seen that the $\mathrm{RuO}_{\mathrm{x}}$ reduction profiles correlate well with the profile of the EUV beam, which implies that reduction is activated by the EUVinduced plasma.

Under conditions that are similar to those expected during EUVL, only the ruthenium in the topmost surface (less than monolayer of oxide) is restored quickly, while the underlying oxide is not reduced. Moreover, the dependence on total ion dose is very weak, as the reduction efficiency falls off after approx. 0.5 monolayer of oxide is reduced. On the other hand, the correlation between the spatial profile of the EUV spot and the spatial profile of the oxide reduction

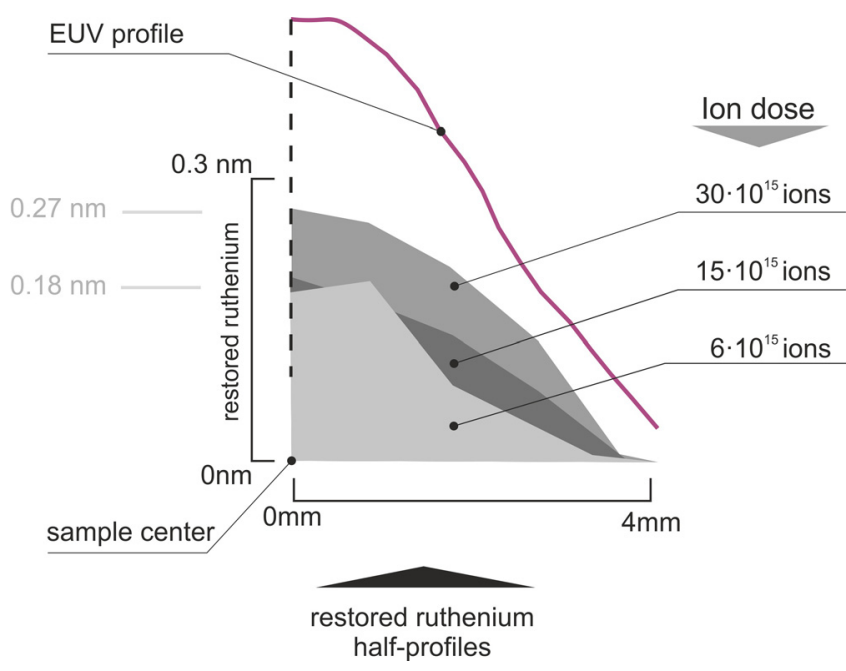

FIG. 11. Ruthenium reduction half-profiles for different ion doses (exposure time). Hydrogen ion flux profile depends on the EUV intensity profile. The dependency between the reduction and the spatial profile of the EUV beam is clearly observable.

shows that there is a clear dependence between $\mathrm{RuO}_{2}$ reduction and ion flux.

Removing underlying layers of oxide requires that hydrogen penetrates, and that the oxygen can diffuse to the surface. For ion energies in the range used here, the stopping distance is estimated to be $1-2 \mathrm{~nm}$, which is sufficient to reduce the entire oxide layer. Therefore, we conclude that the reduction of ruthenium is limited by the diffusion of oxygen to the surface.

\section{DISCUSSION}

The results of plasma experiments show that there are two different mechanisms (process- 1 and process-2) responsible for the reduction of ruthenium oxide. In what follows, we propose that process- 1 may follow a similar reaction pathway to that for atomic hydrogen, while process- 2 may involve direct vacancy generation that leads to oxygen removal.

Process-1 involves low energy ions that are likely to quickly neutralize at or very close to the surface. Thus, the reduction processes may be similar to that found for hydrogen atoms, which is described in Ref. 10. In this case, oxide removal from a $\mathrm{RuO}_{2}$ surface (likely to be the predominant oxide in our case ${ }^{13}$ ) is described by a stepwise process. In a $\mathrm{RuO}_{2}$ matrix, hydrogen may be present within the lattice, weakly bound to the $\mathrm{O}_{\text {lattice }}$ and on the surface, adsorbed to the bridge, $\mathrm{O}_{\text {bridge, }}$, between two ruthenium atoms. According to Ref. 10, the surface of the ruthenium oxide hydrogenates through adsorption. In addition, hydrogen atoms, adsorbed at surface $\mathrm{O}_{\text {bridge }}$ sites, form hydroxyl groups that are chemically bound to the surface $\left(\mathrm{O}_{\text {bridge }}-\mathrm{H}\right)$. The formation of the $\mathrm{OH}$ bond results in a nearby vacancy

$$
2\left(\mathrm{O}_{\text {bridge }}-\mathrm{H}\right) \rightarrow\left(\mathrm{O}_{\text {absorb }}-2 \mathrm{H}\right)+\mathrm{O}_{\text {bridge }}+\text { surface vacancy. }
$$

This is followed by water desorption. As a result, oxygen and vacancy concentration gradients form in the oxide film. These gradients are perpendicular to the surface and lead to 
oxygen diffusion from the oxide bulk under the following scheme: ${ }^{10}$

$$
\mathrm{O}_{\text {lattice }}+\text { surface vacancy } \rightarrow \mathrm{O}_{\text {bridge }}+\text { vacancy in the lattice. }
$$

As a result, oxygen escapes to the surface, where it can be removed as described earlier.

In process-2, the ions have sufficient energy to directly generate vacancies, rather than by hydrogen exchange and water desorption. According to earlier research, vacancies have an important (if not a crucial) role in metal recovery from oxides under the action of hydrogen. In particular, oxygen bonds are broken at the surface with the resulting formation of the hydroxyl group under the action of a high-energy ion. This, in turn, leads to the formation of oxygen vacancies in the surface layer. Nonetheless, low ion fluxes may not provide sufficient ions to allow the reaction between hydroxyl groups and hydrogen (either as an ion or a radical) to proceed at high efficiency. Furthermore, at ion energies above $50 \mathrm{eV}$, a significant portion of the flux penetrates the surface and is unable to reduce surface oxide. Thus, process-2 may also depend on a combined flux of ions and radicals to provide both the energy to activate hydroxyl formation and a supply of reactant. With increased radical concentrations, there is a high probability for the hydroxyl group to react with a radical to form water. Figures 8 and 9 show that this is related to the increased reduction efficiency in the presence of hydrogen atoms on ruthenium oxide.

Once the surface is reduced, further $\mathrm{RuO}_{2}$ reduction is rate limited by two processes: the implantation or diffusion of reactive hydrogen species and the diffusion of oxygen. This naturally leads to a deceleration of the efficiency of oxygen removal from deeper layers.

\section{CONCLUSIONS}

Our experiments show that the top atomic layer of ruthenium can be effectively and efficiently reduced from an oxide to a metallic state under hydrogen ion fluxes that are relevant to EUVL. Furthermore, we show that the balance between reduction and oxidation can be effectively controlled by adjusting the partial pressure of hydrogen in the chamber.

Additional experiments demonstrate that the removal of oxide from beneath the surface is more difficult to describe. Rather high energy ions are required to deliver reactive hydrogen species to the buried oxide, while the rate at which oxygen diffuses to the surface is found to be very low. As a result, the reduction rate is most likely limited by the slow diffusion of oxygen through ruthenium.

\section{APPENDIX: ACTINOMETRY OF H ATOMS ON Ar}

The density of hydrogen atoms produced by $13.56 \mathrm{MHz}$ ICP discharge over the samples surfaces in the quartz tube was measured by actinometry. For a low-pressure plasma, when the dissociation degree is low, the hydrogen dissociation degree can be estimated from the ratio of emission lines of $\mathrm{H}$ atoms and stable actinometer atoms like any of the noble gases. We use Ar, a common choice. The ratio of atomic hydrogen to molecular hydrogen is given by

$$
\frac{[H]}{\left[H_{2}\right]} \approx C_{A r}^{H} \frac{I_{H}}{I_{A r}} \zeta
$$

where $\zeta=\frac{[A r]}{\left[H_{2}\right]}, C_{A r}^{H}=\frac{S_{\lambda m^{A r}}^{A r} \lambda_{i j}^{H}}{S_{\lambda_{i j}}^{H} \lambda_{n m}^{A \theta}} \frac{k_{e}^{A r}}{k_{e}^{H}}=A \cdot \frac{A_{e}^{A r}}{k_{e}^{H}}, A$ is some constant, and $S_{\lambda}$ is the sensitivity of the detection system at the wavelength $\lambda . k_{e}{ }^{H}$ and $k_{e}{ }^{A r}$ are excitation rate constants of the emitting states of $\mathrm{H}$ and $\mathrm{Ar}$ atoms. The rate constants of excitation by direct electron impact $k_{e}{ }^{H}$ and $k e^{A r}$ are very sensitive to energy distribution function (EEDF). However, the ratio of the excitation rate constants is insensitive to changes to the EEDF, as long as the electron temperature, $T_{e}$, exceeds the difference of atom excitation energies. This condition is fulfilled for the given experiments described here. Thus, the Maxwellian EEDF with the measured $\mathrm{T}_{\mathrm{e}}$ and respective excitation cross sections were used to calculate the ratio $k_{e}{ }^{A r} / k_{e}{ }^{H}$.

To perform the experiments, $10 \%$ of $\mathrm{Ar}$ was added to hydrogen and the difference in ratio $I_{H} / I_{A r}$ of intensities of $\mathrm{H}_{\alpha}(656 \mathrm{~nm})$ to $\operatorname{Ar}\left(2 \mathrm{p}_{1}\right)(750 \mathrm{~nm})$ emission lines in the $40 \mathrm{MHz}$ SWD plasma column was measured. By switching the $13.56 \mathrm{MHz}$ ICP discharge on and off, the additional atomic hydrogen contributed by the ICP was measured. The detection system was placed such that the atomic hydrogen concentrations were measured over the different sample positions. The emission spectrum of $40 \mathrm{MHz}$ SWD discharge in $10 \% \mathrm{Ar} / \mathrm{H}_{2}$ mixture is shown at the bottom of Fig. 12 . The difference of this spectrum with the spectrum when the 13.56 MHz ICP discharge was switched on is shown at the top of Fig. 12. The increase in atomic hydrogen is calculated from the difference between ICP on and ICP off spectra.

The hydrogen dissociation degree is estimated from actinometric signal, i.e., the ratio $I_{H} / I_{A r} . I_{H}$ is intensity of $\mathrm{H}_{\alpha}$ line from the differential spectrum (top of Fig. 12), and $I_{A r}$ is intensity of $\operatorname{Ar}\left(2 p_{1}\right)$ line (bottom Fig. 12) from the usual spectrum. Figure 13 shows the ratio of $I_{H} / I_{A r}$ as a function of the distance along the quartz tube. The hydrogen dissociation degree is rather small, and falls fast along the tube. The low

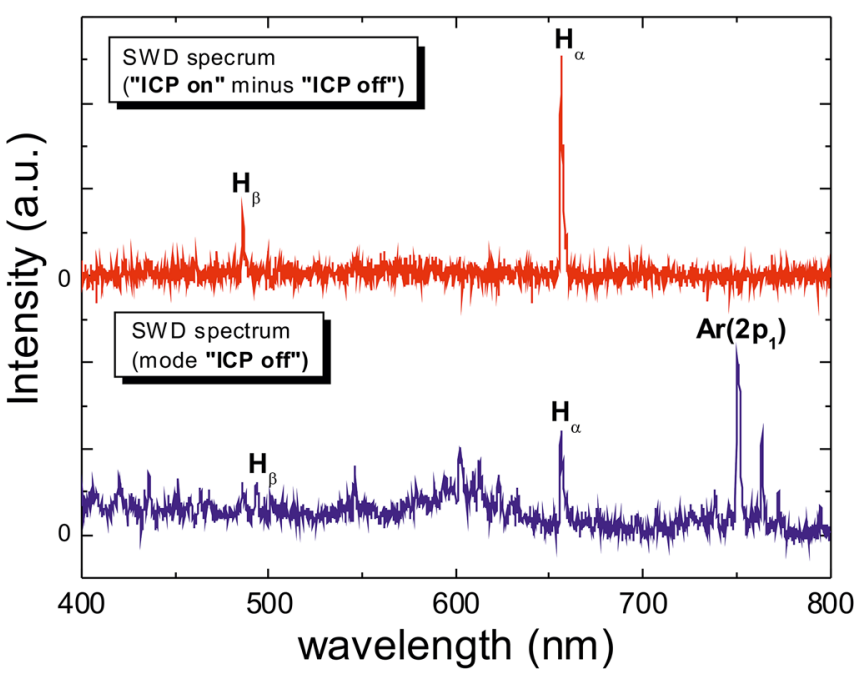

FIG. 12. Bottom blue curve is emission spectrum of the $40 \mathrm{MHz}$ SWD discharge $(10 \mathrm{~W})$ in $10 \% \mathrm{Ar} / \mathrm{H}_{2}$ mixture $(100 \mathrm{mTorr})$ with the $13.56 \mathrm{MHz}$ ICP discharge switched off. Top red curve is the difference between the $40 \mathrm{MHz}$ SWD emission spectra when the $13.56 \mathrm{MHz}$ ICP discharge was switched on and off (only emission of $\mathrm{H}$ atoms produced by ICP is observed). 


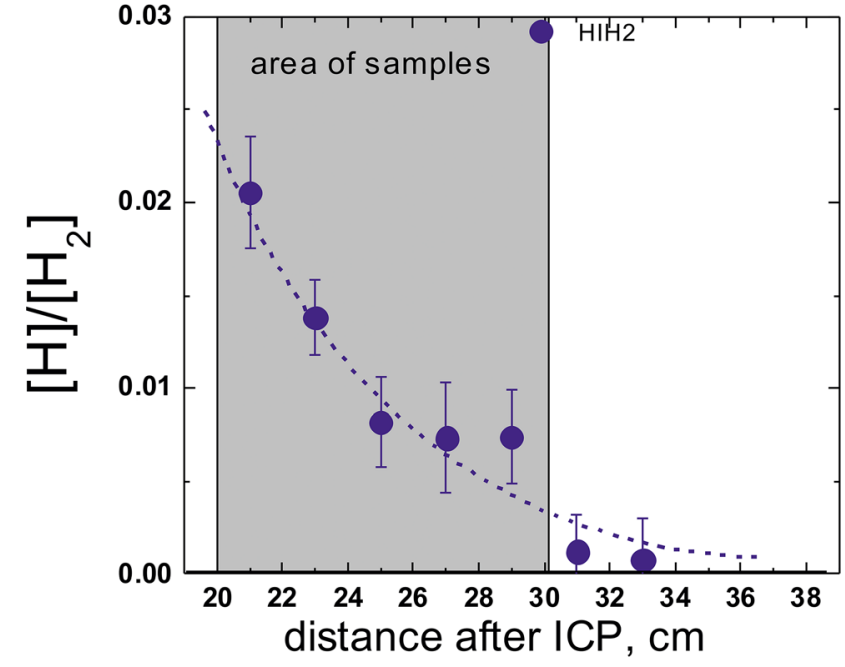

FIG. 13. Dissociation degree $[\mathrm{H}] /\left[\mathrm{H}_{2}\right]$ in the afterglow of $13.56 \mathrm{MHz}$ ICP discharge (100 mTorr 10\% Ar:90\% $\left.\mathrm{H}_{2}, 10 \mathrm{~W}\right)$. The distance range to the samples is shown by grey rectangle.

atomic hydrogen concentration is due to the fast $\mathrm{H}$ atom recombination on open surfaces of metallic flanges between the ICP (which is $\sim 45 \%$ efficient) and tube.

${ }^{1}$ See http://www.itrs.net for International Technology Roadmap for Semiconductors.

${ }^{2}$ V. Banine and J. Benschop, Proc. SPIE 5401, 7861 (2004).

${ }^{3}$ A. Al-Ajlony, A. Kanjilal, M. Catalfano, S. S. Harilal, A. Hassanein, and B. Rice, Proc. SPIE 8322, 832232 (2012).

${ }^{4}$ J. Q. Chen, E. Louis, C. J. Lee, H. Wormeester, R. Kunze, H. Schmidt, D. Schneider, R. Moors, W. van Schaik, M. Lubomska, and F. Bijkerk, Opt. Express 17, 16969-16979 (2009).
${ }^{5}$ T. E. Madey, N. S. Faradzhev, B. V. Yakshinskiy, and N. V. Edwards, Appl. Surf. Sci. 253, 1691-1708 (2006).

${ }^{6}$ J. Hollenshead and L. Klebanoff, J. Vac. Sci. Technol. B 24, 64-82 (2006).

${ }^{7}$ S. Matsunari, T. Aoki, K. Murakami, Y. Gomei, S. Terashima, H. Takase, M. Tanabe, Y. Watanabe, Y. Kakutani, M. Niibe, and Y. Fukuda, Proc. SPIE 6517, 65172X (2007).

${ }^{8}$ M. Niibe, K. Koida, and Y. Kakutani, J. Vac. Sci. Technol. B 29, 011030 (2011).

${ }^{9}$ H. Oizumi, A. Izumi, K. Motai, I. Nishiyama, and A. Namiki, Jpn. J Appl. Phys., Part 2 46, L633-L635 (2007).

${ }^{10}$ D. Ugur, A. J. Storm, R. Verberk, J. C. Brouwer, and W. G. Sloof, Microelectron. Eng. 110, 60-65 (2013).

${ }^{11}$ F. Brecelj and M. Mozetic, Vacuum 40(1-2), 177-181 (1990).

${ }^{12}$ Y. Sawada, H. Tamaru, M. Kogoma, M. Kawase, and K. Hashimoto, J. Phys. D: Appl. Phys. 29, 2539 (1996).

${ }^{13}$ Y. Iwasaki, A. Izumi, H. Tsurumaki, A. Namiki, H. Oizumi, and I. Nishiyama, Appl. Surf. Sci. 253, 8699-8704 (2007).

${ }^{14}$ R. M. van der Horst, J. Beckers, E. A. Osorio, T. H. M. van de Ven, and V. Y. Banine, Plasma Sources Sci. Technol. 24(6), 065016 (2015).

${ }^{15}$ D. I. Astakhov, W. J. Goedheer, C. J. Lee, V. V. Ivanov, V. M. Krivtsun, K. N. Koshelev, D. V. Lopaev, R. M. van der Horst, J. Beckers, E. A. Osorio, and F. Bijkerk, J. Phys. D: Appl. Phys. 49(29), 295204 (2016).

${ }^{16}$ A. Dolgov, D. Lopaev, T. Rachimova, A. Kovalev, A. Vasil'eva, C. J. Lee, V. M. Krivtsun et al., J. Phys. D: Appl. Phys. 47, 065205 (2014).

${ }^{17}$ F. Liu, M. Sturm, C. J. Lee, and F. Bijkerk, Surf. Sci. 646, 101-107 (2016).

${ }^{18}$ J. Hollenshead and L. Klebanoff, J. Vac. Sci. Technol. B 24, 118 (2006).

${ }^{19}$ Handbook of Optical Constants of Solids, edited by E. D. Palik (Elsevier Inc., 1997), ISBN: 978-0-12-544415-6.

${ }^{20}$ Y. M. Chung, E. M. Lee, T. Masuoka, and J. A. R. Samson, J. Chem. Phys. 99, 885 (1993).

${ }^{21}$ A. Dolgov, D. Lopaev, C. J. Lee, E. Zoethout, V. Medvedev, and O. Yakushev, Appl. Surf. Sci. 353, 708-713 (2015).

${ }^{22}$ S. M. Zyryanov, A. S. Kovalev, D. V. Lopaev et al., Plasma Phys. Rep. 37, 881 (2011).

${ }^{23}$ A. Dolgov, O. Yakushev, A. Abrikosov, E. Snegirev, V. M. Krivtsun, and C. J. Lee, Plasma Sources Sci. Technol. 24(3), 035003 (2015). 